ARTICLE

\title{
A mycorrhizae-like gene regulates stem cell and gametophore development in mosses
}

\author{
Shuanghua Wang ${ }^{1,2}$, Yanlong Guan ${ }^{1}$, Qia Wang ${ }^{1}$, Jinjie Zhao ${ }^{1}$, Guiling Sun (1) ${ }^{3}$, Xiangyang Hu ${ }^{4}$, Mark P. Running ${ }^{5}$,
} Hang Sun ${ }^{1 凶} \&$ Jinling Huang (1D $1,3,6 \bowtie$

Plant colonization of land has been intimately associated with mycorrhizae or mycorrhizaelike fungi. Despite the pivotal role of fungi in plant adaptation, it remains unclear whether and how gene acquisition following fungal interaction might have affected the development of land plants. Here we report a macro2 domain gene in bryophytes that is likely derived from Mucoromycota, a group that includes some mycorrhizae-like fungi found in the earliest land plants. Experimental and transcriptomic evidence suggests that this macro2 domain gene in the moss Physcomitrella patens, PpMACRO2, is important in epigenetic modification, stem cell function, cell reprogramming and other processes. Gene knockout and over-expression of PpMACRO2 significantly change the number and size of gametophores. These findings provide insights into the role of fungal association and the ancestral gene repertoire in the early evolution of land plants.

\footnotetext{
${ }^{1}$ Key Laboratory for Plant Diversity and Biogeography of East Asia, Yunnan Key Laboratory for Fungal Diversity and Green Development, Kunming Institute of Botany, Chinese Academy of Sciences, Kunming 650201, China. ${ }^{2}$ University of Chinese Academy of Sciences, Beijing 100049, China. ${ }^{3}$ State Key Laboratory of Crop Stress Adaptation and Improvement, Key Laboratory of Plant Stress Biology, School of Life Sciences, Henan University, Kaifeng 475001, China. ${ }^{4}$ Shanghai Key Laboratory of Bio-Energy Crops, School of Life Sciences, Shanghai University, Shanghai 200444, China. ${ }^{5}$ Department of Biology, University of Louisville, Louisville, KY, USA. ${ }^{6}$ Department of Biology, East Carolina University, Greenville, NC, USA. ${ }^{凶}$ email: sunhang@mail.kib.ac.cn; huangj@ecu.edu
} 
milestone in the evolution of plants is their conquest of land, a feat accomplished through a partnership with fungi, most commonly mycorrhizae-like association ${ }^{1,2}$. The dramatic habitat transition from water to land posed tremendous challenges for plants, including desiccation, increased UV irradiation and temperature fluctuation, and exposure to novel pathogens ${ }^{3,4}$. In response, early land plants evolved various strategies and features to adapt to hostile terrestrial environments ${ }^{5,6}$. In addition to their many physiological innovations, land plants underwent major developmental changes, notably three-dimensional growth as well as alternation of gametophyte and sporophyte generations ${ }^{7,8}$. Such developmental changes allowed a more complex body plan and morphological diversity in land plants, which would in turn enhance their reproductive success, survival, and niche expansion ${ }^{7-9}$.

Understanding the adaptative strategies in early land plants requires a complete picture of their underlying molecular mechanisms. Bryophytes (liverworts, hornworts, and mosses) include extant members of early-diverging land plant lineages ${ }^{10,11}$. In addition to their important systematic position, bryophytes have retained many structures and functions, including genes and pathways, that were inherited from ancestral land plants 9,12 . Some of these genes or pathways might have been lost secondarily from other land plants over time, but they are still found in bryophytes. These ancestral genes and pathways, together with those inherited from charophytes (the closest relatives of land plants), may not only provide critical insights into the adaptive strategies of plants during their transition from water to land ${ }^{5,6,13}$, but also the sequence of evolutionary changes that occurred when land plants became increasingly complex ${ }^{14}$, both structurally and physiologically. Nevertheless, compared to flowering plants, bryophyte model organisms, such as the moss Physcomitrella patens and the liverwort Marchantia polymorpha, remain largely understudied 15,16 . Frequently, knowledge of the physiological and developmental processes in bryophytes heavily relies on studies on homologous genes and processes in flowering plants (e.g., Arabidopsis thaliana). This approach of evolutionary development has identified some core components of key pathways conserved throughout land plant evolution, but, on the other hand, has provided little information on the toolkit specific to early land plants. For instance, several genes, including Defective Kernel 1, APB-like and CLAVATA-like genes, are known to be conserved in stem cell functions and threedimensional growth between bryophytes and flowering plants $^{17-19}$. Nevertheless, how other ancestral genes or pathway components (e.g., those only retained in basal land plants) contributed to stem cell development and three-dimensional growth of early land plants remains obscure.

Macrodomains are known for their capability of binding or cleaving ADP-ribose from cellular molecules and, therefore, play a key role in ADP-ribosylation, an important post-translation modification mechanism that is yet to be fully elucidated ${ }^{20-22}$. They regulate a number of cellular activities, such as chromatin modification, transcription and translation, DNA repair, and cell differentiation ${ }^{20,22}$. Within the macrodomain superfamily, the macro2 family (Pfam 14519) is the least studied and its biological functions are largely unclear ${ }^{20}$. In this study, we report a macro2 domain gene in bryophytes that was likely acquired by the ancestral land plant from mycorrhizae-like fungi. This mycorrhizae-like macro2 domain gene has been lost secondarily from vascular plants but is still retained in bryophytes. Experimental evidence shows that the macro2 domain gene in $P$. patens (PpMACRO2) regulates multiple key processes, including the development of stem cells and gametophores (the dominant form of three-dimensional growth in bryophytes), cell reprogramming and tissue regeneration, as well as epigenetic changes. We speculate that $P p M A C R O 2$ is involved in histone modification through ADP-ribosylation, which in turn triggers additional epigenetic changes through other mechanisms in $P$. patens. We further discuss the adaptive role of the macro2 domain and ancestral gene repertoire in land plants.

\section{Results}

Mycorrhizae-like fungal origin of land plant macro2 gene. The PpMACRO2 protein includes a single macro2 domain of 145 aa, with an additional N-terminus of 97 aa and a short C-terminus of about 64 aa. With the PpMACRO2 protein sequence (Genbank accession number: XP_024388278) as query, we performed a BLAST search of the NCBI non-redundant $(n r)$ protein sequence database, the 1000 plants project (OneKP) and other resources, including the recently published genomes of hornworts (Anthoceros angustus), ferns (Azolla filiculoides and Salvinia cucullata) and charophytes (e.g., Chara braunii, Spirogloea muscicola, Mesotaenium endlicherianum, Mesostigma viride, and Chlorokybus atmophyticus) ${ }^{23-28}$, as well as our internal draft genome of another charophyte, Interfilum paradoxum. Additional pHMMER search was performed against Reference Proteomes. Our searches provided hits only from mosses, liverworts, fungi, bacteria, viruses, and a few other eukaryotes (E-value cutoff $=1 \mathrm{e}$ 6) (Supplementary Figs. 1 and 2). Many of the bacterial hits are annotated as phage tail proteins. No hits could be detected in hornworts and vascular plants in our analyses. Additionally, although a BLAST search of OneKP and NCBI dbEST databases, which only contain transcriptomic data, yielded hits from green algal species, no hit could be identified from any complete genome of green algae that was generated from axenic cultures. Similarly, although hits were found from the charophyte Spirogyra pratensis in NCBI dbEST database, our polymerase chain reaction (PCR) from genomic DNA failed to amplify sequences from an unspecified congeneric species Spirogyra sp. (Supplementary Fig. 3 and Supplementary Table 1). Therefore, the possibility that these green algal hits are from foreign sources (i.e., contamination) cannot be excluded.

Notably, moss and liverwort macro2 protein sequences not only had the highest coverage (up to 78\%) and percent identity (up to 59\%) with hits from Mucoromycota fungi (Rhizophagus irregularis, Gigaspora rosea, Jimgerdemannia flammicorona, etc.), they also uniquely shared several amino acid residues (Fig. 1a and Supplementary Fig. 5a). Consistent with the sequence comparison, phylogenetic analyses showed that PpMACRO2 and its homologs from other mosses, liverworts and fungi (mostly Mucoromycota fungi) formed a well-supported clade, which in turn grouped with other fungal sequences (Supplementary Figs. 4 and $5 b)$.

PpMACRO2 protein is localized in both nucleus and cytoplasm. The PpMACRO2 gene is located on chromosome 11 of $P$. patens and annotated to include 1499 nucleotides according to Phytozome (phytozome.jgi.doe.gov/pz/portal.html). Although the annotated PpMACRO2 gene consists of three introns and four exons, our reverse transcription PCR (RT-PCR) experiments were only able to recover exons 1,3 , and 4 , without the annotated exon 2 (Fig. 1b). This result is consistent with the Phytozome annotation that the transcription level of exon 2 is minimal (Supplementary Fig. 6). The RT-PCR cloned sequence is predicted to contain an intact macro2 domain (Supplementary Fig. 7).

To visualize the subcellular localization of PpMACRO2, we performed transient protoplast transformation of the PpMACRO2 gene. The coding sequence of PpMACRO2 was tagged with enhanced green fluorescent protein (EGFP) and transformed using polyethylene glycol (PEG)-mediated protoplast transient 
a

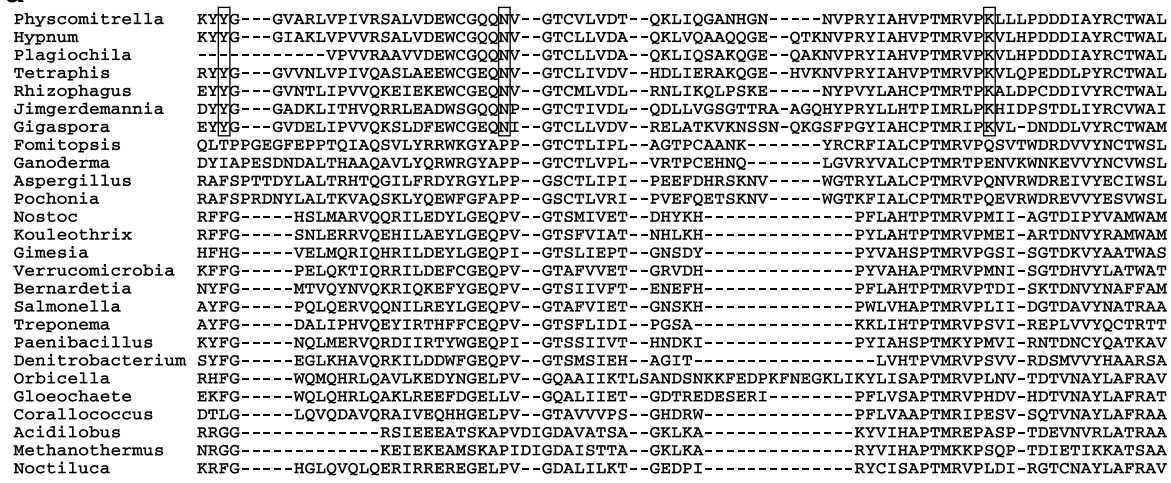

b

-

KRFG--- - HGLOVOLOERIRKEAMSKAPIDIGDAISTTA- -GKLKA -

RYVIHAPTMKKPSQP-TDIETIKKATSA

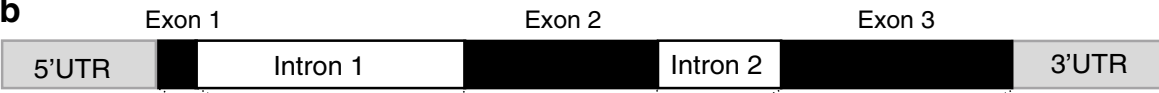

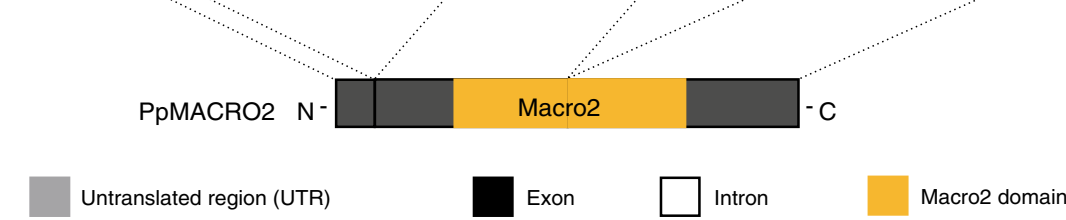

MKLNELVAAWQDEITRELPPALLDQSSTATSPISIATYNDFIQEFLKSTQVDCIVSPANSFGLMDGGLDLTI SKYYGGVARLVPIVRSALVDEWCGQQNVGTCVLVDTQKLIQGANHGNNVPRYIAHVPTMRVPKLLLP DDDIAYRCTWALLTAVRKHNNEILKQDQTRTGGERINTVLCSGFGTGIGNFPLQECARQMVLAVKHFA

ERTSSNSTNEDDLRGSSHLMTLWDKAERIEDAVCCVVPEKSGETPSSPCTFEVLGK*

Fig. 1 PpMACRO2 sequence relationship and gene annotation. a Multiple alignment for PpMACRO2 protein sequence and homologs sampled from different major groups. Boxes in the alignment show amino acids uniquely shared by bryophytes and Mucoromycota fungi. Detailed molecular phylogeny of PpMACRO2 and homologs is shown in Supplementary Figs. 4 and 5. $\mathbf{b}$ Schematic diagram of PpMACRO2 gene structure and encoded protein sequence based on RT-PCR and sequencing evidence. Exons 1, 2, and 3 correspond to exons 1, 3, and 4, respectively, of the P. patens v3.3 annotation in Phytozome.

expression in P. patens. GFP fluorescence was observed in both the nucleus and cytoplasm (Supplementary Fig. 8).

PpMACRO2 promotes protonema growth and gametophore budding. To understand the functions of $P$ PMACRO2, we generated four knockout $(k o)$ and three over-expression (OE) genetically modified plants of PpMACRO2, respectively. The ko and $O E$ plants were characterized by genotyping the transformants using genomic PCR, real-time quantitative RT-PCR (qRTPCR), and Southern blotting (Supplementary Figs. 9 and 10). All $k o$ and $O E$ plants were haploids based on flow cytometric analyses (Supplementary Fig. 11). The wild-type (WT), ko, and $O E$ plants were cultivated under normal growth conditions, and their phenotypes were observed and measured. Compared to WT plants of $P$. patens, the ko mutants produced smaller protonemata, but larger and fewer gametophores; the reverse was true for $O E$ lines (Figs. 2 and $3 \mathrm{a}-\mathrm{d}$ ). Specifically, the average number of gametophores in ko mutants decreased by about $29 \%$ relative to WT plants of $P$. patens, whereas the number of gametophytes increased by $33-71 \%$ in the three different $O E$ lines (Fig. 3c). Furthermore, the ko mutants also produced longer gametophores (Fig. 2d).

To further understand the role of PpMACRO2 in gametophore development, we generated transgenic plants of $P$. patens in which EGFP and $\beta$-glucuronidase (GUS) reporter genes were inserted inframe immediately before the stop codon of PpMA$\mathrm{CRO} 2$ via homologous recombination (Supplementary Fig. 12). The resulting plants expressed a PpMACRO2-EGFP-GUS fusion protein under the control of its native promoter within the endogenous genomic environment. The PpMACRO2pro: PpMACRO2-EGFP-GUS lines had no visible developmental differences compared to WT plants. Fluorescent signal of the fusion protein was detected during the development of gametophores in P. patens. Notably, the signal was particularly strong in developing buds and apical meristems of leafy gametophores (Fig. 3e, f and Supplementary Fig. 13). This evidence, together with changes in the number and size of gametophores in ko and $O E$ plants (Figs. 2 and $3 \mathrm{a}-\mathrm{d}$ ), suggests that $P$ PMACRO2 plays a key role in gametophore development of $P$. patens.

It has been known that cytokinin induces gametophore bud formation, and over-budding can be obtained by exogenous application of cytokinin ${ }^{29,30}$. To investigate the interplay between PpMACRO2 and cytokinin in bud formation, we treated $P$. patens WT plants and PPMACRO2 ko mutants with $1 \mu \mathrm{moll}^{-1} 6$ benzylaminopurine (BA), a synthetic cytokinin. Under normal growth conditions, the ko mutants generated about $30 \%$ fewer gametophores than WT plants. Under 6-BA treatment, the number of gametophores in the ko mutants, however, was only about $12 \%$ fewer compared with the wild type (Fig. 3d), indicating that cytokinin could partially rescue PpMACRO2 ko mutants in gametophore budding.

PpMACRO2 in stem cell development and cell reprogramming. To assess whether PpMACRO2 participates in other developmental processes in $P$. patens, we performed GUS histochemical assays using PpMACRO2pro:PpMACRO2-EGFP-GUS. GUS expression was consistently detected in stem cells throughout the lifecycle of $P$. patens (Fig. 4). Upon germination of spores and throughout the protonema phase, GUS staining was detected in both chloronemata and caulonemata, but clearly stronger in apical and side-branch stem cells (Fig. 4a-c). During the development of gametophores, strong GUS staining was 
a

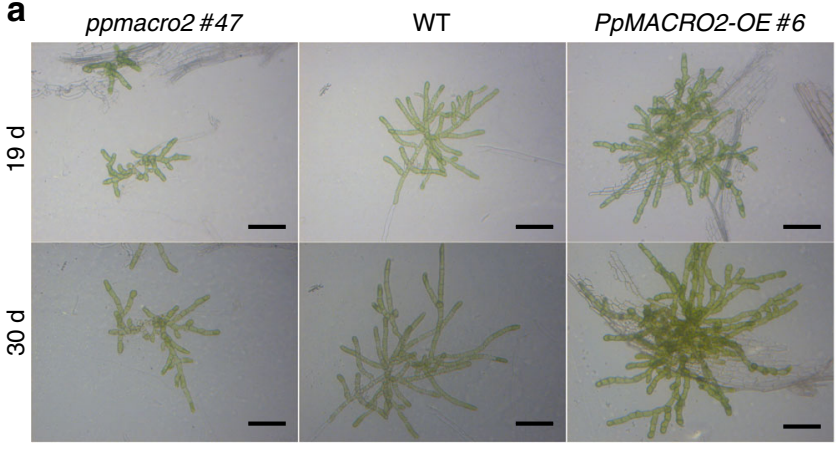

b

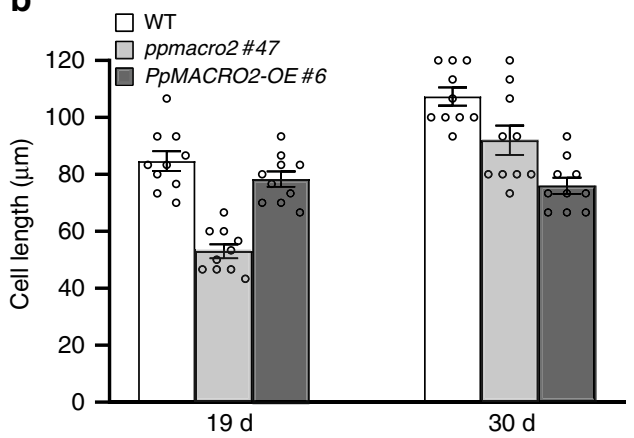

C

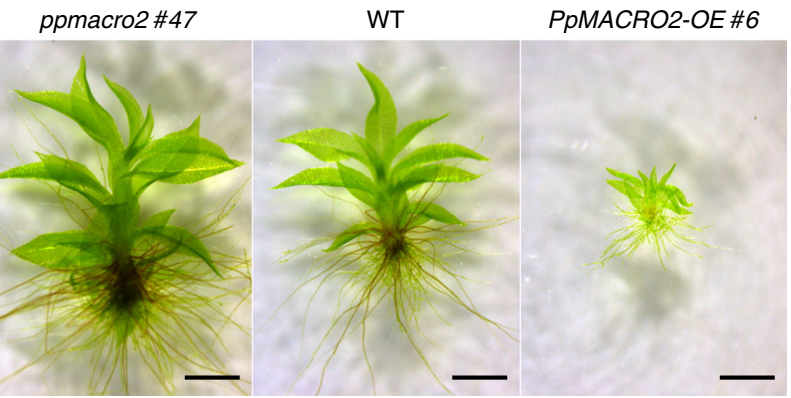

d

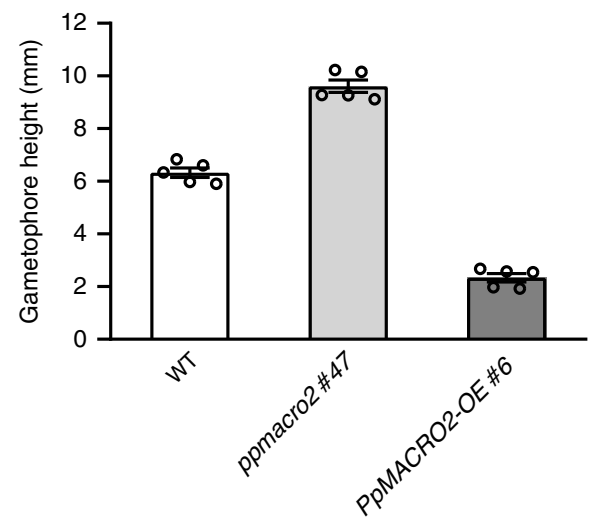

Fig. 2 PpMACRO2 affects sizes of protonemata and gametophores in P. patens. a, b Protonemal cell growth and elongation in WT, ko and OE plants. Protonemal cell length in ko mutants decreased by about 40\% than in the wild type after 19 days, but there was no significant difference between $O E$ lines and the wild type. After 30 days, protonemal cells in ko mutants were only about $15 \%$ shorter compared to the wild type, whereas protonemal cells of $O E$ lines were about $30 \%$ shorter. These results show that protonemata grow much faster in ko mutants but slower in OE lines compared to the wild type between day 19 and day 30. Micrograph images shown were observed from at least three biological replicates. Data show means \pm s.e.m. of ten biological replicates for WT and mutant plants. c, $\mathbf{d}$ Sizes of gametophores developed from four-week-old WT, ko, and OE plants. Gametophore heights were measured twice independently, with similar results. Micrograph images provided were observed from five biological replicates. Data show means \pm s.e.m. of five biological replicates. Scale bar: $200 \mu \mathrm{m}$ in a; $500 \mu \mathrm{m}$ in c.

mostly restricted to the three-faced bud apical cells and leafy apical stem cells (Fig. 4d, e). Before fertilization, GUS activity was detected in developing archegonia (Fig. 4f), where staining was stronger in unfertilized eggs, whereas no GUS staining was detected in antheridia. After fertilization, GUS signal was detectable in mature sporophytes and individual spores (Fig. 4g, h). The above observation suggests that PpMACRO2 is involved in stem cell development in $P$. patens.

Given the strong expression of $P p M A C R O 2$ in apical stem cells of both protonemata and gametophores, we investigated whether and how PPMACRO2 might affect cell reprogramming and regeneration. Protoplast regeneration was first performed for WT, $k o$ and $O E$ plants, respectively. During the first two days after protoplast isolation, cells divided more quickly in $O E$ lines than in WT and ko plants (Fig. 5a). After five days, more cells formed in $O E$ lines compared to the wild type, whereas cells were fewer and shorter in ko mutants, consistent with the smaller protonemata observed in the early propagation stage of ko mutants (Fig. 2a, b). Between day 5 and day 10, branching of protonemata was faster in $O E$ lines, whereas it was slower in ko mutants, relative to WT plants. On day 13, we observed bud initiation in $O E$ lines, but not in WT and ko plants (Fig. 5a). Protonema branching became faster and protonema cells were longer in $k o$ mutants after 13 days, but fewer gametophores were produced compared to WT and $O E$ plants.

Physcomitrella patens tissues are capable of regeneration when damaged $^{31}$. To understand the role of PpMACRO2 in tissue regeneration, we detached leaves from gametophores of WT, $k o$ and $O E$ plants, respectively, and cultivated them on $\mathrm{BCD}$ medium. Approximately $70 \mathrm{~h}$ after detachment, protonema filaments began to emerge in WT plants. During the same growth period, more filaments were observed from the $O E$ lines, whereas none was formed in the ko mutants (Fig. 5b). To visualize the expression of $P$ PMACRO2 during tissue regeneration, we performed parallel experiments using PpMACRO2EGFP-GUS knockin lines and examined GUS staining and GFP fluorescence on the detached leaves (Fig. $5 \mathrm{c}$ and Supplementary Fig. 14). The GUS expression pattern in WT plants of $P$. patens was similar to that previously reported for PpFIE, which encodes a component of Polycomb group complex in stem cell maintenance ${ }^{32}$. At $48 \mathrm{~h}$ after detachment, GUS staining and GFP fluorescence became visible on the surface of detached leaves (Fig. 5c). Protonema filaments then developed gradually from the GUS-stained and GFP-fluoresced cells. These data indicate that $P p M A C R O 2$ promotes cell reprogramming and tissue regeneration in P. patens.

PpMACRO2 affects epigenetics and transcription factors. Stem cell and gametophore development involves a number of activities $^{30,33}$. To understand how PpMACRO2 may affect other activities, we generated RNA-seq data for both $k o$ and $O E$ lines of $P p M A C R O 2$, and then identified differentially expressed genes relative to the WT plants. Notably among the differentially 
a
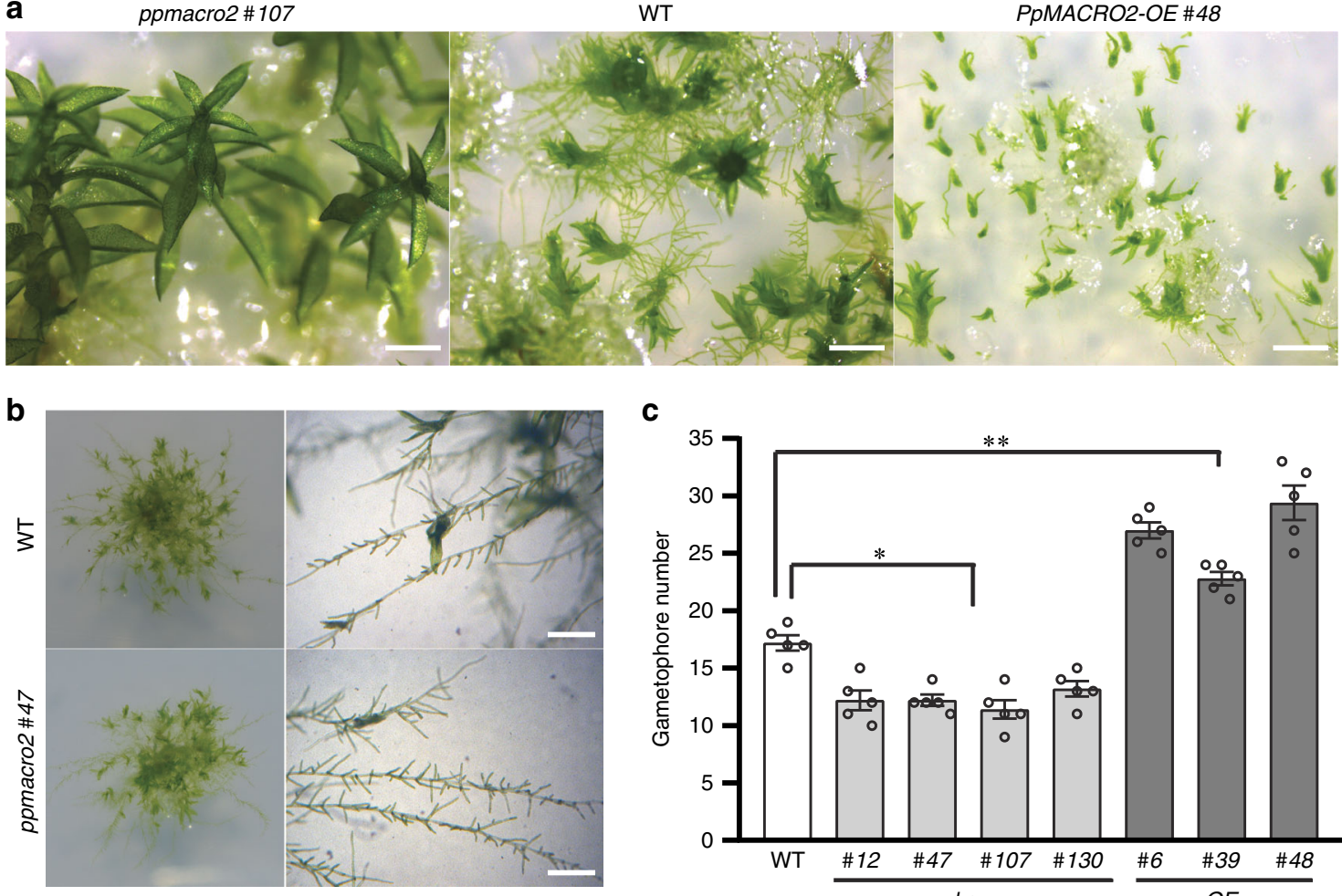

C
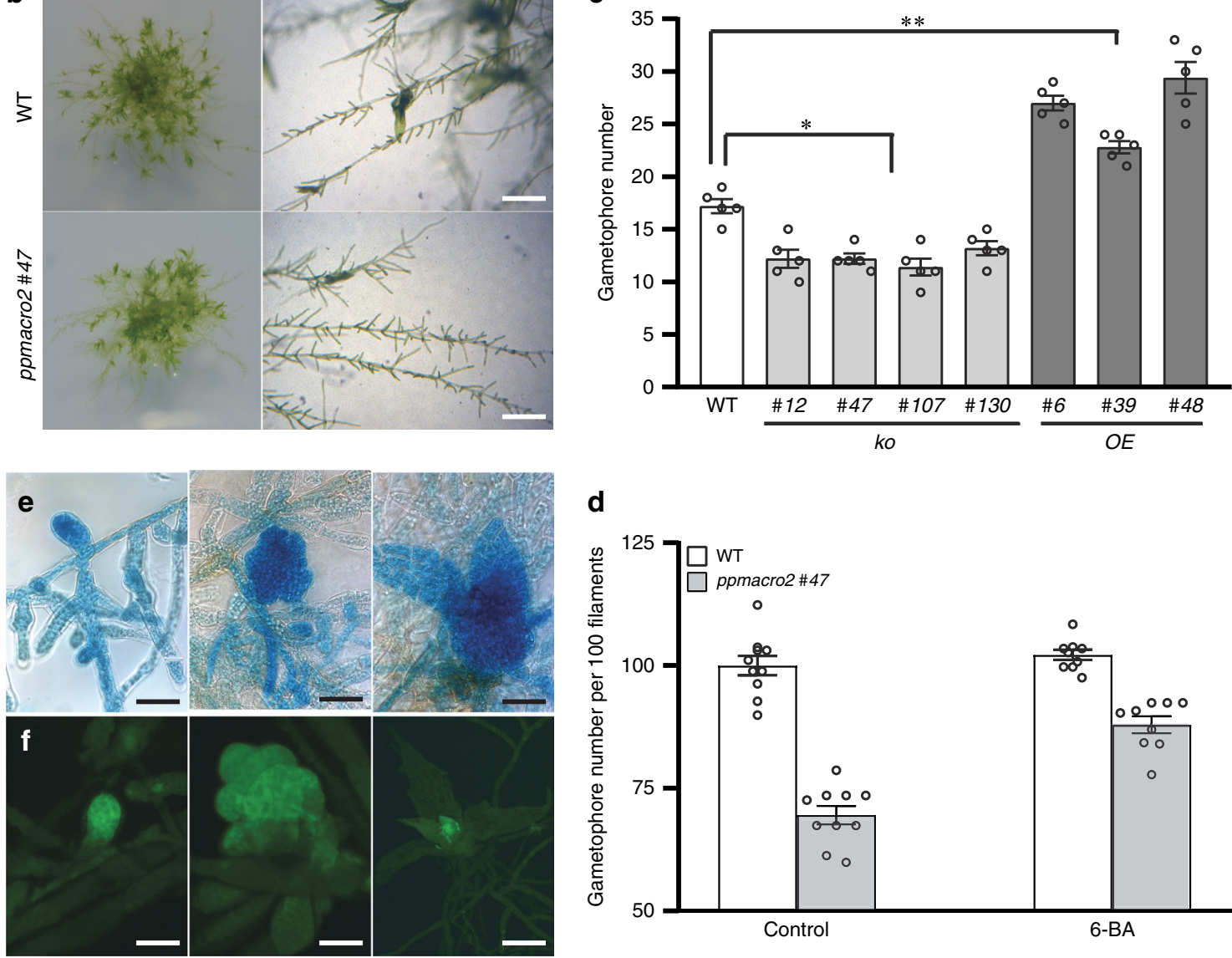

d

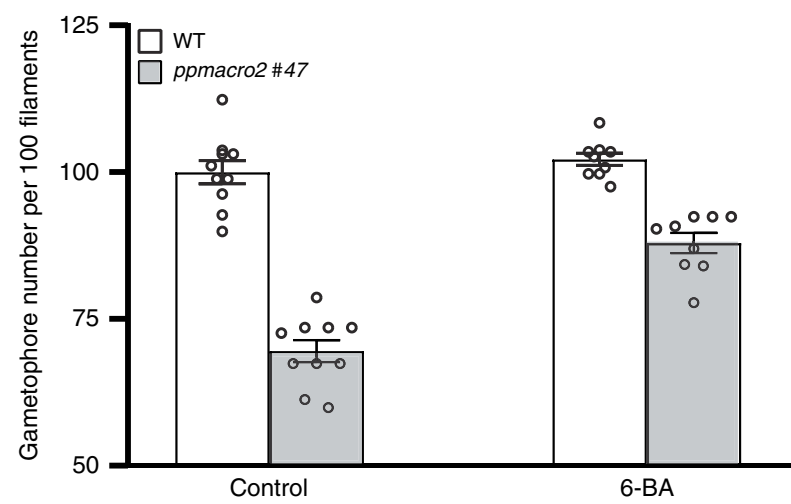

Fig. 3 PpMACRO2 affects the number of gametophores developed in P. patens. a, c Number of gametophores developed from four-week-old WT, ko and OE plants. Seven-day-old protonemata of WT, ko and OE plants were harvested and suspended with sterile water, respectively, and then crushed. The same amount of suspension $\left(\mathrm{OD}_{600}=0.4\right)$ was propagated onto $\mathrm{BCD}$ medium and grown for about four weeks. Gametophore numbers were counted under microscope's field of view. Micrograph images given were observed from five biological replicates. Data show means \pm s.e.m. of five biological replicates for WT and mutant plants. Asterisks indicate a statistically significant difference compared with WT plants based on a two-tailed Student's $t$-test $\left({ }^{\star} p<0.05\right.$, $\left.{ }^{\star \star} p<0.01\right)$. b, d Number of gametophores per filament in WT and ko plants of PpMACRO2. Protonemata of approximately $1 \mathrm{~mm}$ in diameter were transplanted onto BCD medium from the wild type and ppmacro2 \#47 respectively, and cultivated for three weeks. Ten clones were counted for the wild type and ko mutant, respectively. Under normal growth conditions, the ko mutant generated about 30\% fewer gametophores than the wild type. However, under $1 \mu \mathrm{mol} \mathrm{I}^{-1}$ 6-BA treatment, the number of gametophores in the ko mutant was only about $12 \%$ fewer compared to the wild type. Micrograph images provided were observed from ten biological replicates. Data show means \pm s.e.m. of ten biological replicates. e, $\mathbf{f}$ GUS staining and green fluorescence of gametophore development. Left to right show a swollen gametophore apical cell, a gametophore with multiple cells, and a leafy gametophore, respectively. Micrograph images shown were observed from at least three biological replicates. Scale bars: $500 \mu \mathrm{m}$ in a, b; $50 \mu \mathrm{m}$ in e, $\mathbf{f}$.

expressed genes are those related to epigenetic modification (Fig. 6 and Supplementary Fig. 15). These genes encode at least four SET domain proteins or putative histone-lysine methyltransferases (Pp3c13_4470, Pp3c13_19810, Pp3c1_8530, and Pp3c17_14770), a homolog of Sin-associated protein 30 (SAP30) (Pp3c20_6230), and a homolog of methyl-CpG binding domaincontaining protein 9 (MBD9) (Pp3c5_19640). In line with the transcriptomic data, our searches of the STRING database predicted protein-protein interaction of PpMACRO2 with histones $\mathrm{H} 2 \mathrm{~A}$ and H2B (Supplementary Fig. 16) ${ }^{34}$. Furthermore, several families of developmental transcription factors were also differentially expressed in PpMACRO2 ko and $O E$ lines. In particular, $A P 2$ and homeobox genes were down-regulated in ko mutants and, conversely, up-regulated in $O E$ lines (Fig. 6a). These 

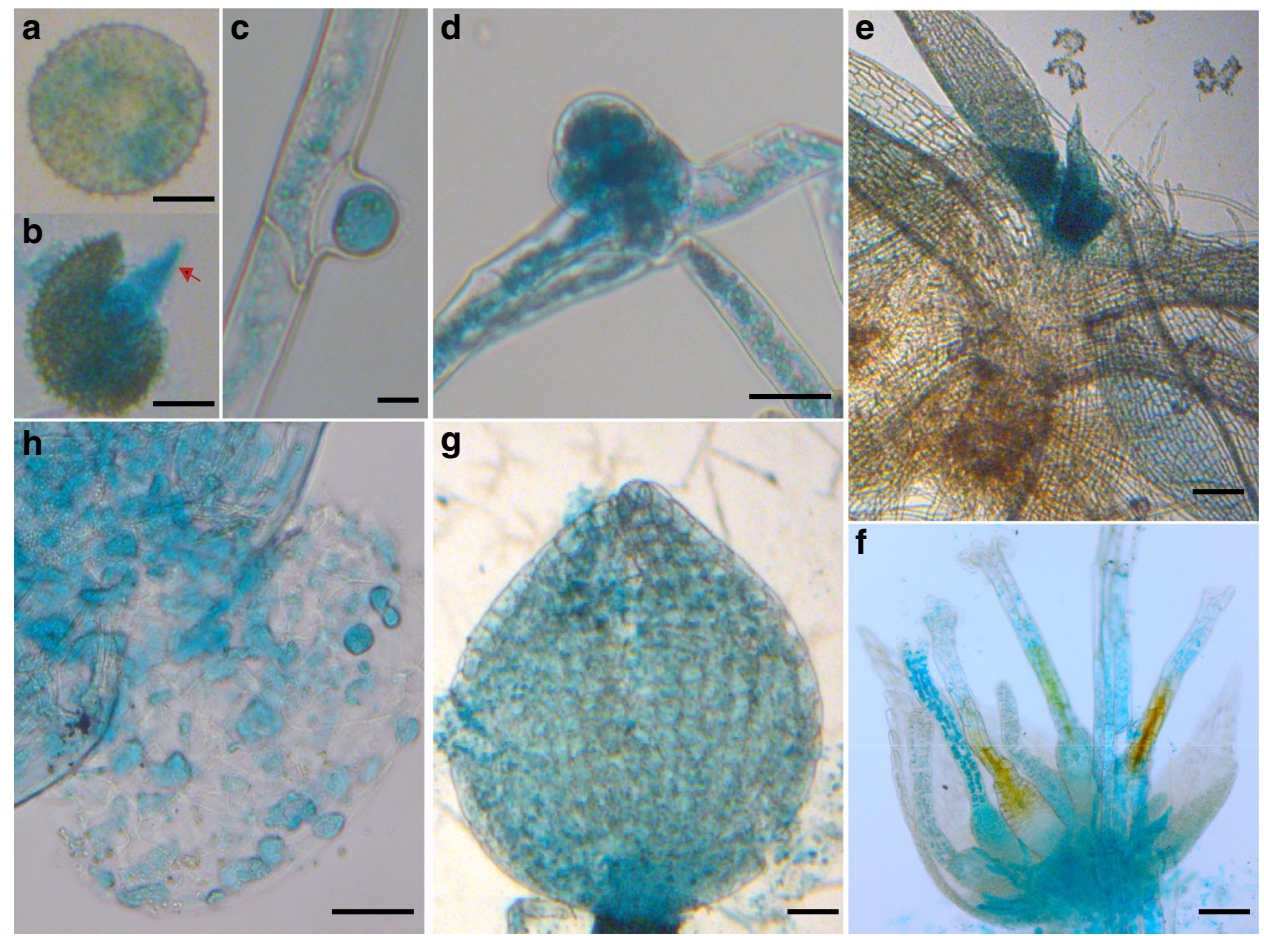

Fig. 4 Expression profile of PPMACRO2 determined by histochemical GUS assays. a Spores. b Germinating spores, with the red arrow indicating the chloronema apical stem cell. c Protonema side-branch apical stem cell. d Juvenile gametophore. e Leafy gametophore. $\mathbf{f}$ Developing archegonium, with GUS activity detected in the egg cell as well as in the archegonia tissue. $\mathbf{g}$ Sporangium. $\mathbf{h}$ Individual spores within sporangium. Micrograph images provided were observed from at least three biological replicates. Scale bars: $25 \mu \mathrm{m}$ in $\mathbf{a}, \mathbf{b} ; 100 \mu \mathrm{m}$ in $\mathbf{c}, \mathbf{d}, \mathbf{f}-\mathbf{h} ; 500 \mu \mathrm{m}$ in $\mathbf{e}$.
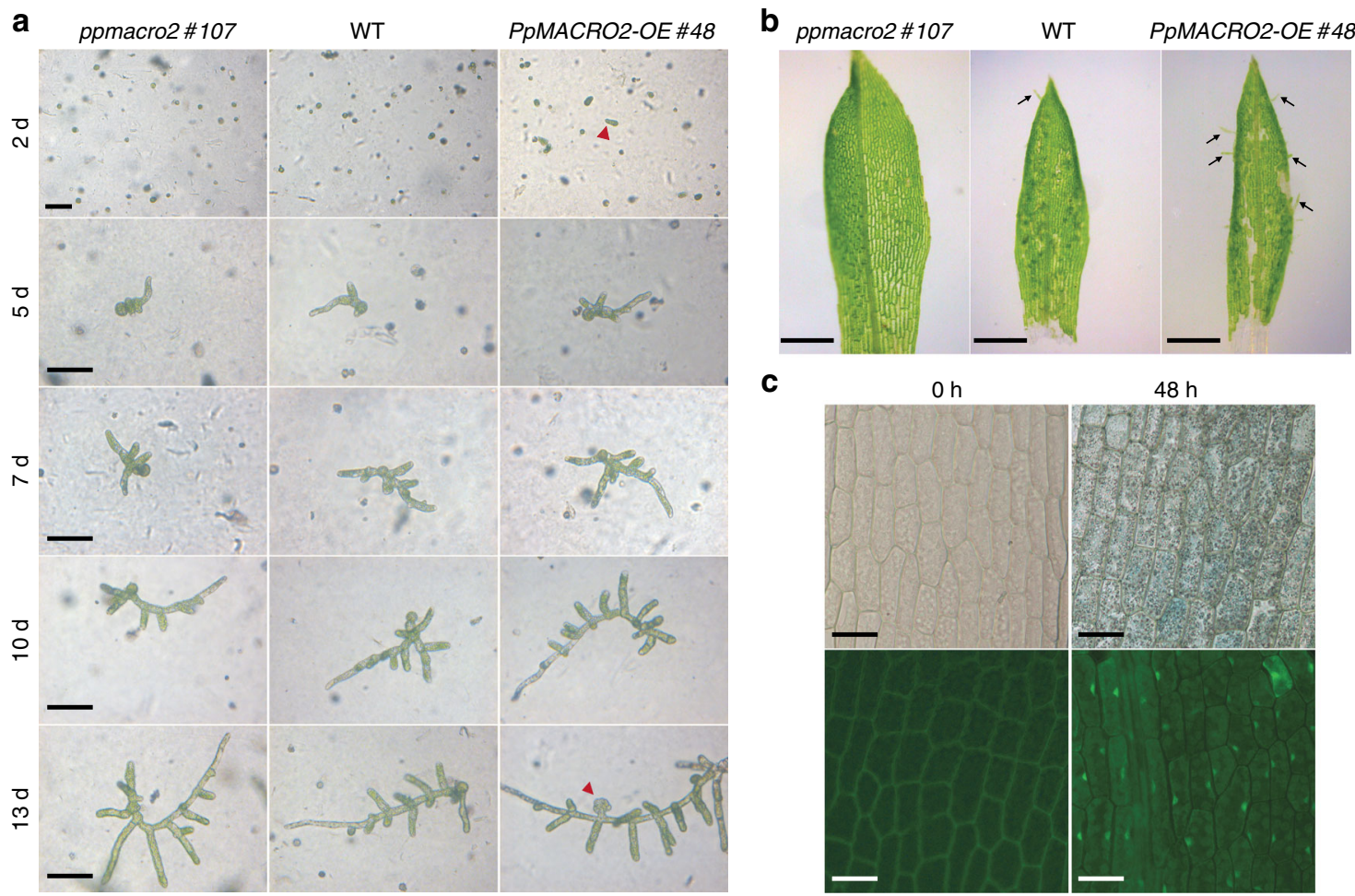

C
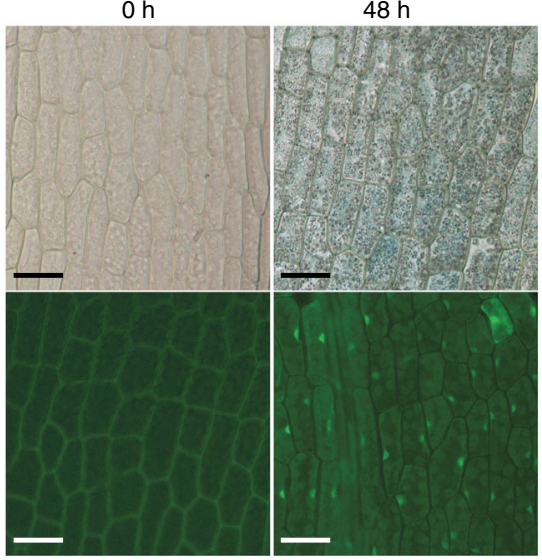

Fig. 5 PpMACRO2 promotes cell reprogramming in P. patens. a Protoplast regeneration, with red arrowheads indicating differentiated cells and buds. b Tissue regeneration using detached leaves from WT plants, as well as ko and OE mutants of $P$. patens. Black arrows indicate emerged protonema filaments. c GUS staining and GFP fluorescence of leaves were observed from PpMACRO2-EGFP-GUS transgenic lines after being detached for $48 \mathrm{~h}$. Micrograph images shown were observed from five biological replicates. Scale bars: $100 \mu \mathrm{m}$ in $\mathbf{a}, \mathbf{c} ; 500 \mu \mathrm{m}$ in $\mathbf{b}$. 
$\mathbf{a}$

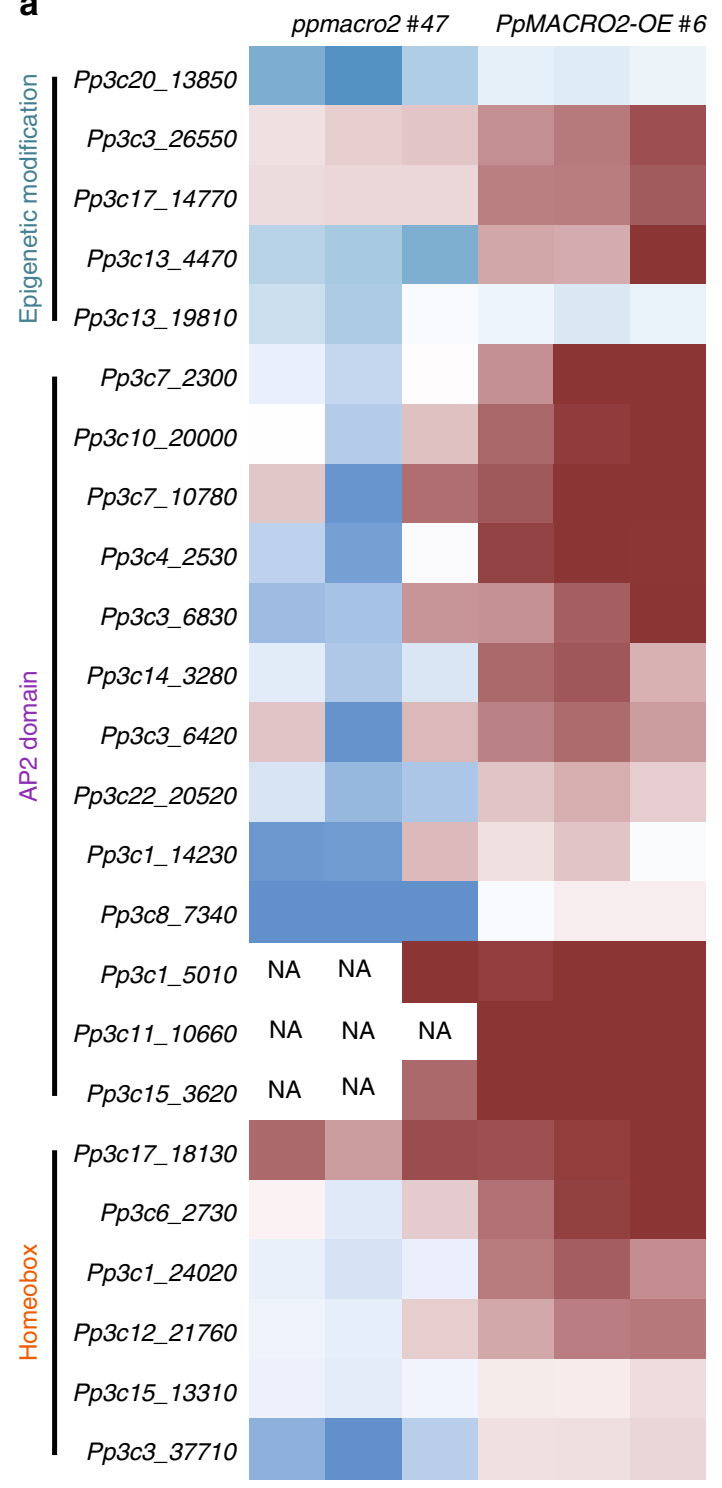

b
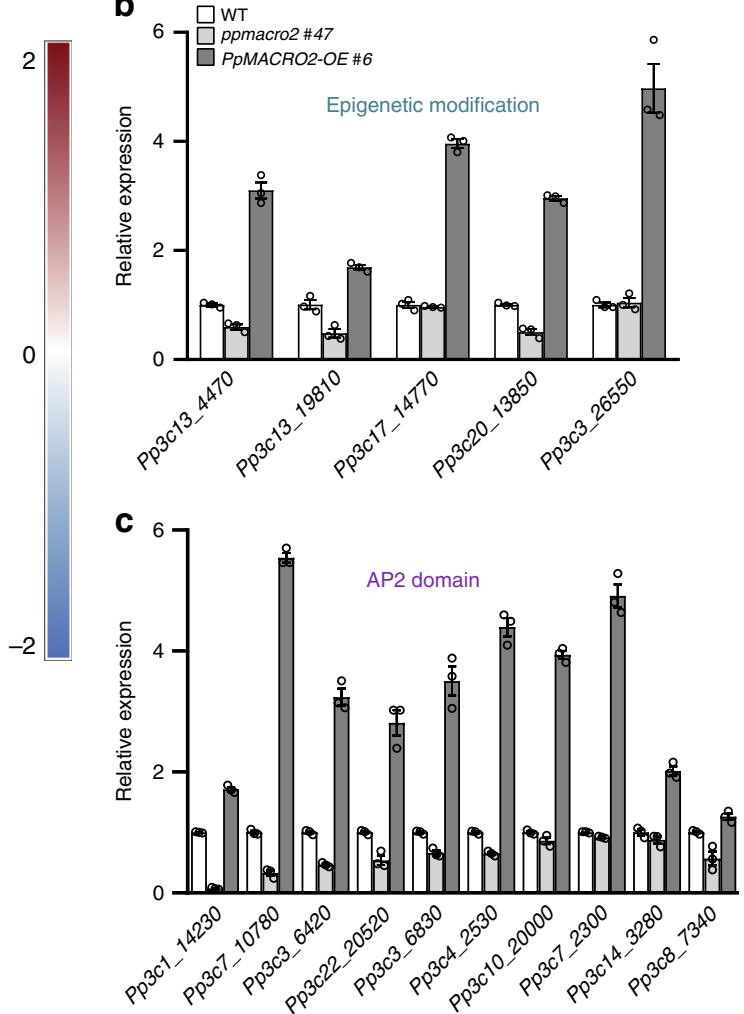

d

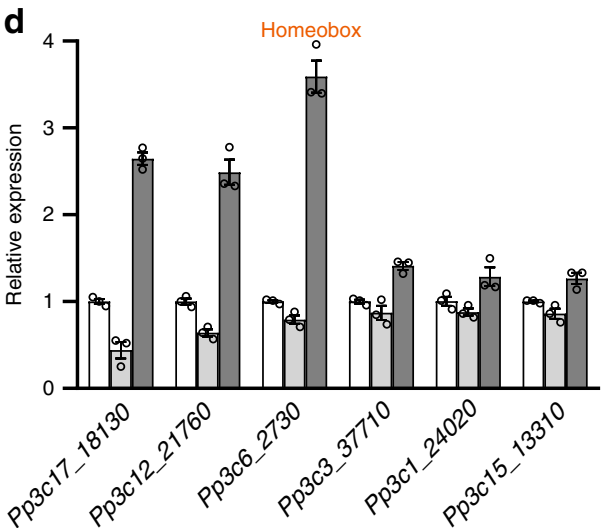

Fig. 6 Differentially expressed genes related to epigenetic modification and stem cell development in $\boldsymbol{k}$ o and $\boldsymbol{O E}$ lines of $\boldsymbol{P}$. patens. a Transcriptional profile from RNA-seq data for a subset of genes related to epigenetic modification and developmental transcription factors in WT plants, as well as ko and OE mutants. Total RNA extracted from WT plants and mutants was used for RNA-seq analyses. b-d Transcript abundance of the subjected genes was confirmed through qRT-PCR with three independent biological replicates, normalized to PpEF1a. Phytozome identifier is used for each gene, and additional information for each gene is provided in Supplementary Table 3. Data show means \pm s.e.m.

transcription factors are often linked to stem cell development and cell reprogramming ${ }^{35}$. For instance, AP2-type and AP2-like transcription factors not only determine stem cell identity and gametophore formation, but also induce cell reprogramming in $P$. patens $s^{17,36}$. Homeobox genes are essential for the development of apical meristems in plants ${ }^{37,38}$. Other noteworthy and differentially expressed genes in $k o$ and $O E$ plants included those related to cell wall formation (e.g., xyloglucan endo-transglycosylase, pectinesterase) and cell division (kinesin-like protein, tubulin beta, and protein regulator of cytokinesis 1), which were often down-regulated in both $k o$ and $O E$ lines (Supplementary Table 2).

To validate the expression pattern from RNA-seq data, we performed qRT-PCR experiments on a subset of the above genes, particularly the genes related to epigenetic modification and developmental transcription factors. RNA-seq expression data for a majority of selected genes were confirmed (Fig. 6b-d and Supplementary Table 3). These data support the role of PpMACRO2 in epigenetic modification, stem cell development, and related processes.

\section{Discussion}

Information on ancestral physiological and developmental pathways in early land plants provides unique insights into the strategies or toolkits adopted by plants during their transition from water to land. Bryophytes retain many features evolved in early land plants and thus provide a living laboratory to understand these pathways and related adaptive strategies ${ }^{9,12}$. In this study, we report a macrodomain gene, PpMACRO2, that is distributed among bryophytes but absent from vascular plants. We show that 
PpMACRO2 is important in stem cell development, cell reprogramming, protonema development, gametophore budding and other processes in P. patens. Considering its universal expression throughout major developmental phases in $P$. patens and its presence in many other bryophytes, it is likely that PPMACRO2 played a fundamental role in the cellular activity and gametophore development during the early evolution of land plants.

Macrodomains are structurally conserved and often functionally related to ADP-ribosylation ${ }^{20,39}$. Because of their distribution in viruses and all three major domains of life (bacteria, archaea, and eukaryotes), macrodomains are believed to be ancient and critical in miscellaneous cellular processes, though the number of macrodomains identified thus far remains limited ${ }^{22}$. Most of the identified macrodomain families (or classes) are well correlated with their functional roles, but little is known about the macro2 family ${ }^{20}$. In plants, although several macrodomain-containing genes have been reported in $A$. thaliana ${ }^{20,40}$, only two poly(ADPribose) glycohydrolase genes (PARGs), which are structurally conserved among eukaryotes, have been functionally investigated $^{41-43}$. To our knowledge, no macro2 domain gene has been reported in plants thus far.

Our data provide a glimpse into the functions and mechanisms of the macro2 domain in the development of plants and other eukaryotes. In particular, both transcriptomic and qRT-PCR data indicate that several key genes in histone modification are differentially regulated in PpMACRO2 ko and $O E$ lines (Fig. 6a, b and Supplementary Fig. 15). This evidence strongly suggests that PpMACRO2 is involved in chromatin changes in P. patens. Intriguingly, the above genes are major players of different histone modification mechanisms. For instance, SET domain proteins commonly regulate histone methylation ${ }^{44-46}$, whereas SAP30 is a key component of the Sin3-histone deacetylase (HDAC) complex that regulates histone deacetylation ${ }^{47,48}$. MBD9, on the other hand, has been demonstrated in A. thaliana to modulate development by modifying chromatin structure via histone acetylation and DNA methylation ${ }^{49}$. It is noteworthy here that macrodomain-containing proteins, such as macroH2A1, macroH2A2, amplified in liver cancer 1 (ALC1) and poly(ADPribose) polymerases (PARPs), are known to induce chromatin conformation changes either as a histone component or a chromatin regulator $20,39,50$. We therefore propose that macro2, similar to some other macrodomain families (e.g., macroH2A-like and ALC1-like), is involved in chromatin modification through ADPribosylation, which in turn triggers a cascade of additional chromatin changes via other epigenetic mechanisms (e.g., histone methylation and acetylation). These cross-talks between different mechanisms may fine-tune the regulation of chromatin conformation and, therefore, the activation/repression of downstream transcription factors in stem cell development (e.g., AP2 and homeobox genes) and other processes (Fig. 7) ${ }^{51,52}$. This model is consistent with the observation that PpMACRO2 is expressed in the nucleus in $P$. patens and the fact that PpMACRO2 has a similar expression profile as the PpFIE gene of the Polycomb group complex (Supplementary Figs. 8 and 14), a known player in histone methylation ${ }^{32,53}$.

Given the role of PPMACRO2 in some key developmental processes of $P$. patens, it is puzzling that this gene has apparently been lost in vascular plants. Presumably, such selective retention in bryophytes points to a unique role of the macro2 domain during the transition of green plants from water to terrestrial environments. It is well known that ADP-ribosylation plays a crucial role in plant responses to biotic and abiotic stresses ${ }^{54,55}$. For instance, PARPs and the macrodomain-containing PARGs are involved in stress responses to pathogens and genotoxic agents $^{41,42}$. Conceivably, because of the involvement of macrodomains in ADP-ribosylation, PpMACRO2 (and its homologs in

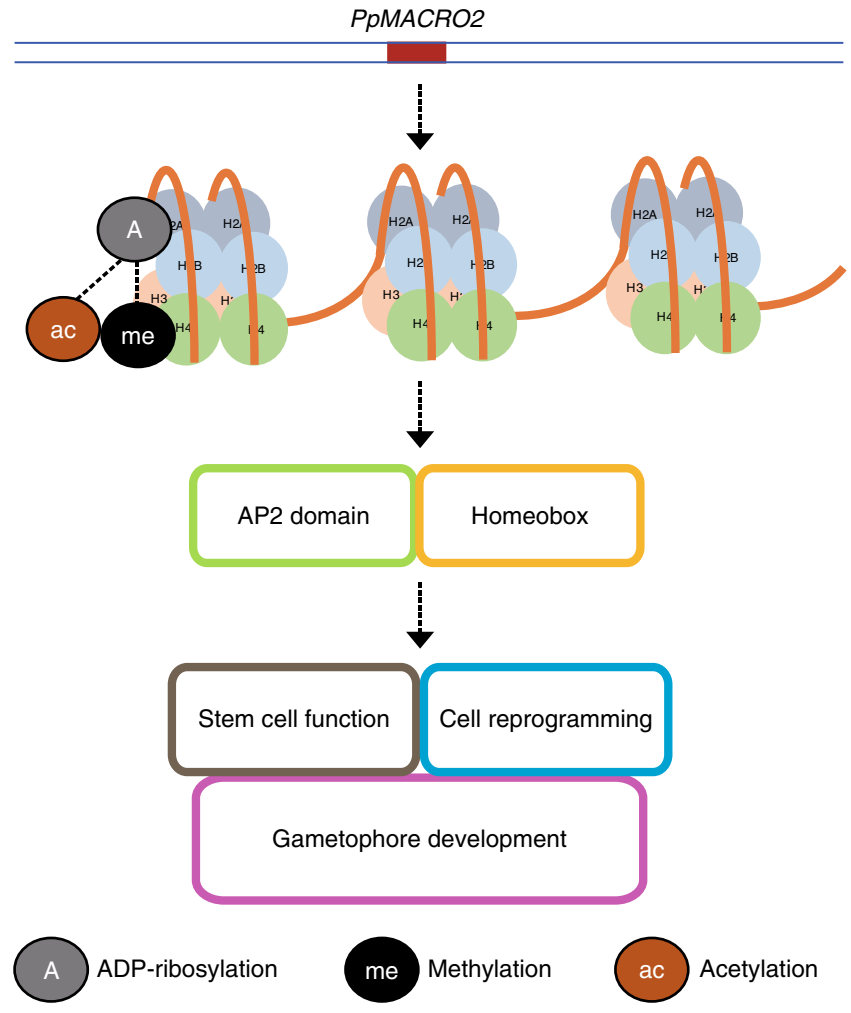

Fig. 7 A proposed model for PpMACRO2 in chromatin modification and development of $\boldsymbol{P}$. patens. PpMACRO2 is likely involved in chromatin modification through ADP-ribosylation, which in turn triggers a cascade of additional chromatin changes via other epigenetic mechanisms, including histone methylation and acetylation. These epigenetic modification mechanisms may fine-tune chromatin conformation, which consequently activates/represses downstream developmental transcription factors (e.g., AP2 and homeobox) and affects stem cell function, cell reprogramming, gametophore development, and other biological processes. Dashed lines indicate that the relationship is less certain.

other bryophytes) might have facilitated the adaptation of early land plants to primeval terrestrial environments (e.g., DNA repair, pathogen resistance), in addition to their developmental role. The retention of this gene may be important for bryophytes, considering their lack of sophisticated protective mechanisms compared to other land plants. In fact, retention of genes in bryophytes (or loss of genes from vascular or seed plants) has been documented in several other cases ${ }^{56-58}$, most of which, to a certain extent, are also related to adaptation of plants to environmental stresses. For instance, actinoporin and hemerythrin genes are involved in desiccation tolerance in mosses, but both appear to have been lost from seed plants ${ }^{56,58}$. ENA ATPase genes, which reportedly confer salt resistance to bryophytes, are also lost from vascular plants ${ }^{57}$. On the other hand, the concurrent loss of PPMACRO2 and other stresses-related genes was also possibly associated with a shift, either structural or physiological, over the course of land plant evolution. It remains to be investigated whether this shift occurred in seedless vascular plants or seed plants.

Other than land plants and fungi, PpMACRO2 homologs are also found in bacteria, viruses, and some other eukaryotes. In bacteria, these sequences are often annotated as phage tail proteins, suggestive of a viral origin (i.e., prophages or remnant phages). Because viruses are commonly found in green algae ${ }^{59-61}$, it is unclear whether the hits in green algal datasets were actually from associated viruses. Intriguingly, both sequence comparison 
and molecular phylogeny showed that PpMACRO2 and other land plant homologs are closely related to sequences from Mucoromycota, a group that includes not only some common mycorrhizal fungi in vascular plants (e.g., Rhizophagus irregularis), but also mycorrhizae-like fungi found in the earliest land plants $^{2,62}$ (Supplementary Figs. 4 and 5). This sequence relatedness between land plants and Mucoromycota might have resulted from several scenarios, including differential gene losses and organellar origin. On the other hand, it is also likely that PPMACRO2 was acquired from mycorrhizae-like fungi by the common ancestor of land plants ${ }^{63}$. This second scenario is not only consistent with the common belief that physical association, such as symbiosis, often facilitates horizontal gene transfer (HGT), but also supported by the amino acid residues uniquely shared by Mucoromycota and bryophyte sequences. Thus far, HGT in eukaryotes remains hotly debated ${ }^{64,65}$ but has been reported in many eukaryotic lineages ${ }^{66-68}$, and foreign genes have also been documented in bryophytes and their charophyte relatives $6,25,27,69$. The vast majority of the documented foreign genes in bryophytes and charophytes, however, have not been investigated experimentally. It merits further detailed studies to understand how organismal interactions and ensuing gene transfer might have impacted the abilities of green plants to colonize land.

\section{Methods}

Phylogenetic and protein-protein interaction analyses. The PpMACRO2 protein sequence (NCBI accession number XP 024388278; Phytozome identifier Pp3c11_23270) was used as query to perform BLASTP searches against NCBI $n r$ protein sequence database, OneKP, the marine microbial eukaryote transcriptome sequencing project (MMETSP), and other relevant databases (e.g., Phytozome, NCBI dbEST, FernBase) (E-value cutoff $=1 \mathrm{e}-6)$. Additional pHMMER searches were performed against References Proteomes (E-value cutoff $=1 \mathrm{e}-6$ ). Representative protein sequences from different lineages were sampled for phylogenetic analyses. Multiple protein sequence alignments were performed using MUSCLE with manual refinement. Gaps and ambiguously aligned sites were removed from alignments. Phylogenetic analyses were performed with a maximum likelihood method using PhyML 3.1 and a distance method using neighbor of PHYLIP 3.695. ModelGenerator was used to determine the optimal model of protein substitution and rate heterogeneity. Bootstrap analyses were performed using 100 replicates.

Protein-protein interaction network analyses for PpMACRO2 were performed using STRING database (https://string-db.org).

Plant materials and culture conditions. The 'Gransden 2004' of $P$. patens was used as WT strain and cultured on BCD and BCDAT media at $25^{\circ} \mathrm{C}$ under $16-\mathrm{h}$ light and 8-h dark regime, light intensity $80 \mu \mathrm{mol}$ photons $\mathrm{m}^{-2} \mathrm{~s}^{-1}$. Protonemata of $P$. patens were grown on BCDAT medium, and gametophores were grown on BCD medium (minus ammonium tartrate).

To count the number of gametophores, 7-day-old protonemata of WT, ko and $O E$ plants were harvested and suspended in $1.5-2 \mathrm{ml}$ of sterile water, respectively, and then crushed. $1 \mathrm{ml}$ of the suspension $\left(\mathrm{OD}_{600}=0.4\right)$ was propagated onto $\mathrm{BCD}$ medium and grown for about four weeks. Gametophore numbers were counted under microscope's field of view. In addition, protonemata of approximately $1 \mathrm{~mm}$ in diameter were transplanted onto BCD medium and cultivated for 3-4 weeks. The number of gametophores was determined by counting gametophores per filament of clone. To obtain sporophytes, protonemata were planted into Jiffy7 (peat moss pot: Jiffy Products International AS, Kristansand, Norway) to grow healthy gametophores. The peat moss pots containing gametophores were submerged in water and moved to $15^{\circ} \mathrm{C}$ under short-day photoperiod (8-hour light and 16-hour dark). Sporophytes began to develop after about 3 weeks of induction under low light and low temperatures. Mature sporangia were collected into a 1.5 $\mathrm{ml}$ microtube and sterilized using $10 \%$ Antiformin for $5 \mathrm{~min}$, and then washed 3 times using sterilized water. Finally, sporangia were crushed in $1 \mathrm{ml}$ sterilized water using the tip of a pipette, and the spore suspension was then mixed gently and poured onto $\mathrm{BCD}$ medium, and grown at $25^{\circ} \mathrm{C}$ under 16-hour light and 8-hour dark photoperiod. The germinated spores were observed and photographed after 3 days of incubation.

Plasmid construction for knockout and over-expression. The vector pTN182 was used to delete PpMACRO2 in WT plants of $P$. patens. Genomic fragments containing the upstream (1007 bp) and the downstream (1270 bp) flanking regions of PpMACRO2 were inserted into the pTN182 vector, respectively. Primers used for plasmid construction are provided in Supplementary Table 4.
The vector pPOG1 was used for over-expression plasmid construction. The complete coding region of PpMACRO2 was amplified from cDNA by RT-PCR using primers based on P. patens v3.3 annotation (https://phytozome.jgi.doe.gov/ $\mathrm{pz} /$ portal.html\#!info?alias=Org_Ppatens) and was subsequently cloned and sequenced. The resulting PpMACRO2 CDS contained $857 \mathrm{bp}$ spliced from 3 exons. $\mathrm{CDS}$ of $P$ PMACRO2 was amplified using primers shown in Supplementary Table 4, and cloned into the PPOG1 vector.

Protoplast transformation. Transformation was performed using the PEGmediated method ${ }^{70} .1 .6 \times 10^{6}$ protoplasts per ml were incubated with about $30 \mu \mathrm{g}$ linearized DNA under PEG treatment, and stable lines were selected by two successive cycles of incubation on nonselective media and selective media (on media containing $20 \mu \mathrm{g} \mathrm{ml}^{-1} \mathrm{G} 418$ or $20 \mu \mathrm{g} \mathrm{ml}^{-1}$ hygromycin). The stable lines were screened using genomic PCR and qRT-PCR. Moreover, Southern blotting was performed to confirm single integration in $k o$ and $O E$ lines. All transgenic lines were examined to be haploid with flow cytometry.

Protein subcellular localization. The vector pM999 was used for plasmid construction of transient expression of PpMACRO2. CDS of PpMACRO2 was amplified by RT-PCR with primers given in Supplementary Table 4 and cloned into the EcoRI-SacI site of pM999, and EGFP was fused to PpMACRO2 for transient expression. Transient transformation was performed using the PEG-mediated method. The transformed protoplasts were incubated in darkness at $25^{\circ} \mathrm{C}$ for $16 \mathrm{~h}$. In addition, the vector pTN85 was used to obtain PpMACRO2pro:PpMACRO2EGFP-GUS stable lines. Genomic fragments containing the upstream (1016 bp) and the downstream (1071 bp) flanking regions of PpMACRO2 were inserted into the pTN85 vector, respectively (Supplementary Fig. 12). Primers used for plasmid construction are provided in Supplementary Table 4. Protoplast transformation was performed and stable lines were screened. Detached leaves of PpMACRO2pro: PPMACRO2-EGFP-GUS lines were cultured on BCD medium for $48 \mathrm{~h}$.

GFP signals were observed for protoplasts and detached leaves. Images were obtained using a microscope (Leica DM5500 B, Germany). The green fluorescence excitation was performed with a $488 \mathrm{~nm}$ Argon laser.

Quantitative RT-PCR. Total RNA was extracted from protonemata of $P$. patens using a RNeasy Plant Mini Kit (Qiagen) and DNase I (Solarbio). cDNA was synthesized using M-MLV reverse transcriptase (Promega M1701). qRT-PCR was performed using Bio-Rad CFX96 Real-Time System (Thermo Fisher Scientific) and TransStart Top Green qRT-PCR kit (Transgen Biotech), with three independent biological replicates. PpEF1 $\alpha$ (elongation factor 1-alpha, Phypa_439314) was used as reference gene to calculate the relative expression.

Genotyping of plant materials. All genetic materials were confirmed using genomic PCR, and the primer sequences are shown in Supplementary Table 5. Products of genomic PCR were detected using gel electrophoresis. qRT-PCR was performed to detect the expression level of $k o$ and $O E$ lines. Primer sequences for qRT-PCR are provided in Supplementary Table 6. In addition, Southern blotting was performed to confirm single integration in $k o$ and $O E$ lines using primers provided in Supplementary Table 7.

Southern blotting. Southern blotting was performed as follows: $\sim 3 \mu \mathrm{g}$ of genomic DNA was digested with restriction enzyme NdeI or BglII (see Supplementary Figs. 9 and 10), run on $0.8 \%(\mathrm{w} / \mathrm{v})$ agarose gel (TransGen Biotech), and transferred to a Hybond-N + nylon membrane (GE Healthcare). Probe labeling, hybridization and detection were performed using Dig High Prime DNA Labeling and Detection Starter Kit II (Roche) according to the supplier's instructions. Primers used for probe amplification are given in Supplementary Table 7.

GUS assay. Plant samples consisting of the PpMACRO2 fused to EGFP and GUS in the WT background were cultivated on BCD medium, and incubated in $20 \mu \mathrm{l} 1 \times$ GUS solution. The histochemical GUS activity was detected using GUS stain kit (Real-Times, China). The GUS reaction mix consisted of the following: $50 \mathrm{mmol}^{-1}$ potassium ferrocyanide, $50 \mathrm{mmol}^{-1}$ potassium ferricyanide, $1 \mathrm{~mol} \mathrm{l}^{-1}$ sodium phosphate buffer, $0.5 \mathrm{~mol} \mathrm{l}^{-1}$ sodium EDTA, $10 \%$ Triton X-100 and water. A separate solution of X-Gluc (5-bromo-4-chloro-3-indolyl-beta-D-glucuronide) at a concentration of $50 \mathrm{mg} \mathrm{X-Gluc} \mathrm{ml}{ }^{-1}$ of N-N dimethylformamide was added to the above reaction mix at a ratio of $20 \mu \mathrm{l}$ of X-Gluc solution to $1 \mathrm{ml}$ of reaction mix The reaction was performed at $37^{\circ} \mathrm{C}$, and reaction time was depending on the tissues examined. Pigments in plant tissues were removed by absolute ethyl alcohol. The stained tissues were observed and photographed using a microscope (Leica DM5500 B, Germany).

Observation of GFP. To observe GFP tagged to PpMACRO2 in the WT background, $P$. patens plant samples, including protoplasts, detached leaves, and gametophores, were prepared and then placed on glass slides. GFP signals of protoplasts, detached leaves, and gametophore shoot apical meristems were observed, and images were obtained using a microscope (Leica DM5500 B, 
Germany). The green fluorescence excitation was performed with a $488 \mathrm{~nm}$ Argon laser.

Transcriptome analyses. Extraction of RNA was performed using a RNeasy Plant Mini Kit (Qiagen) and DNase I (Solarbio) for transcriptome sequencing. RNA-seq libraries were prepared with NEB kit, and sequencing was conducted using a HiSeq $\mathrm{X}$ Ten (Illumina) to obtain $150 \mathrm{bp}$ paired-ends. The generated reads were firstly filtered and then mapped onto the reference genome of $P$. patens from Phytozome (https://phytozome.jgi.doe.gov) using HISAT2. The calculation of gene expression levels was performed with StringTie. Finally, differentially expressed genes of $k o$ and $O E$ lines compared to WT plants were identified using DESeq2.

Chromosomal ploidy analyses. The chromosomal ploidy of WT, ko and OE lines was analyzed, respectively, using BD FacsCalibur (USA) flow cytometer. Protonemata of $P$. patens were treated and incubated with DNA fluorochrome propidium iodide (PI) and the relative fluorescence of the stained nuclei was then measured. The cytometer was equipped with an argon ion laser operating at 488 $\mathrm{nm}$. The PI fluorescence was collected by $620 \mathrm{~nm}$ fluorescence-2 (FL2) filter. Parameters for data acquisition were kept constant for all samples. Sample flow rate was set at about 100 nuclei/s and at least 6000 nuclei were acquired for each sample. The results acquired were later analyzed using Cell Quest software. Densely gathered nuclei region in dot plot was gated and considered for final analysis to avoid unwanted counts. The average of coefficient of variation values (CV) for G1 peaks was used to evaluate the results. The results with $\mathrm{CV}<5 \%$ were considered as reliable. Histograms were analyzed using Modifit 3.0 software.

Reporting Summary. Further information on research design is available in the Nature Research Reporting Summary linked to this article.

\section{Data availability}

RNA-seq data generated as part of the study have been deposited to the NCBI SRA database under the BioProject accession PRJNA615867 [https://www.ncbi.nlm.nih.gov/ bioproject/615867]. The source data underlying Figs. 2-6, and Supplementary Figs 3, 8, $9 \mathrm{~b}-\mathrm{d}, 10 \mathrm{~b}-\mathrm{d}, 13-15$ are provided as a Source Data file. Any other data supporting the findings of this study are available from the corresponding authors upon request.

Received: 22 November 2019; Accepted: 2 April 2020;

Published online: 24 April 2020

\section{References}

1. Martin, F. M., Uroz, S. \& Barker, D. G. Ancestral alliances: plant mutualistic symbioses with fungi and bacteria. Science 356, eead4501 (2017).

2. Field, K. J. \& Pressel, S. Unity in diversity: structural and functional insights into the ancient partnerships between plants and fungi. N. Phytol. 220, 996-1011 (2018).

3. de Vries, S. et al. On plant defense signaling networks and early land plant evolution. Commun. Integr. Biol. 11, 1-14 (2018).

4. Rensing, S. A. Great moments in evolution: the conquest of land by plants. Curr. Opin. Plant Biol. 42, 49-54 (2018).

5. Rensing, S. A. et al. The Physcomitrella genome reveals evolutionary insights into the conquest of land by plants. Science 319, 64-69 (2008).

6. Bowman, J. L. et al. Insights into land plant evolution garnered from the Marchantia polymorpha genome. Cell 171, 287-304.e215 (2017).

7. Pires, N. D. \& Dolan, L. Morphological evolution in land plants: new designs with old genes. Philos. Trans. R. Soc. Lond. B Biol. Sci. 367, 508-518 (2012).

8. Jill Harrison, C. Development and genetics in the evolution of land plant body plans. Philos. Trans. R. Soc. Lond. B Biol. Sci. 372, 20150490 (2017).

9. Ligrone, R., Duckett, J. G. \& Renzaglia, K. S. Major transitions in the evolution of early land plants: a bryological perspective. Ann. Bot. 109, 851-871 (2012).

10. Qiu, Y. L. et al. The deepest divergences in land plants inferred from phylogenomic evidence. Proc. Natl Acad. Sci. USA 103, 15511-15516 (2006).

11. Puttick, M. N. et al. The interrelationships of land plants and the nature of the ancestral embryophyte. Curr. Biol. 28, 733-745.e732 (2018).

12. Renzaglia, K. S., Duff, R. J. T., Nickrent, D. L. \& Garbary, D. J. Vegetative and reproductive innovations of early land plants: implications for a unified phylogeny. Philos. Trans. R. Soc. Lond. B Biol. Sci. 355, 769-793 (2000).

13. de Vries, J. \& Archibald, J. M. Plant evolution: landmarks on the path to terrestrial life. N. Phytol. 217, 1428-1434 (2018).

14. Tomescu, A. M., Wyatt, S. E., Hasebe, M. \& Rothwell, G. W. Early evolution of the vascular plant body plan - the missing mechanisms. Curr. Opin. Plant Biol. 17, 126-136 (2014).

15. Shimamura, M. Marchantia polymorpha: taxonomy, phylogeny and morphology of a model system. Plant Cell Physiol. 57, 230-256 (2016).
16. Ishizaki, K. Evolution of land plants: insights from molecular studies on basal lineages. Biosci. Biotechnol. Biochem. 81, 73-80 (2017).

17. Aoyama, T. et al. AP2-type transcription factors determine stem cell identity in the moss Physcomitrella patens. Development 139, 3120-3129 (2012).

18. Perroud, P. F. et al. Defective Kernel 1 (DEK1) is required for threedimensional growth in Physcomitrella patens. N. Phytol. 203, 794-804 (2014)

19. Whitewoods, C. D. et al. CLAVATA was a genetic novelty for the morphological innovation of 3D growth in land plants. Curr. Biol. 28, 2365-2376.e2365 (2018)

20. Rack, J. G., Perina, D. \& Ahel, I. Macrodomains: structure, function, evolution, and catalytic activities. Annu. Rev. Biochem. 85, 431-454 (2016).

21. Palazzo, L., Mikoc, A. \& Ahel, I. ADP-ribosylation: new facets of an ancient modification. FEBS J. 284, 2932-2946 (2017).

22. Feijs, K. L., Forst, A. H., Verheugd, P. \& Luscher, B. Macrodomain-containing proteins: regulating new intracellular functions of mono(ADP-ribosyl)ation. Nat. Rev. Mol. Cell Biol. 14, 443-451 (2013).

23. Nishiyama, T. et al. The chara genome: secondary complexity and implications for plant terrestrialization. Cell 174, 448-464.e424 (2018).

24. Li, F. W. et al. Fern genomes elucidate land plant evolution and cyanobacterial symbioses. Nat. Plants 4, 460-472 (2018)

25. Cheng, S. et al. Genomes of subaerial zygnematophyceae provide insights into land plant evolution. Cell 179, 1057-1067.e1014 (2019).

26. Wang, S. et al. Genomes of early-diverging streptophyte algae shed light on plant terrestrialization. Nat. Plants 6, 95-106 (2020).

27. Zhang, J. et al. The hornwort genome and early land plant evolution. Nat. Plants 6, 107-118 (2020).

28. Leebens-Mack, J.H. et al. One thousand plant transcriptomes and the phylogenomics of green plants. Nature 574, 679-685 (2019).

29. von Schwartzenberg, K. et al. Cytokinins in the bryophyte Physcomitrella patens: analyses of activity, distribution, and cytokinin oxidase/dehydrogenase overexpression reveal the role of extracellular cytokinins. Plant Physiol. 145, 786-800 (2007).

30. Moody, L. A. The 2D to 3D growth transition in the moss Physcomitrella patens. Curr. Opin. Plant Biol. 47, 88-95 (2019).

31. Cove, D. J. \& Knight, C. D. The moss Physcomitrella patens, a model system with potential for the study of plant reproduction. Plant Cell 5, 1483-1488 (1993)

32. Mosquna, A. et al. Regulation of stem cell maintenance by the Polycomb protein FIE has been conserved during land plant evolution. Development 136, 2433-2444 (2009)

33. Kofuji, R. \& Hasebe, M. Eight types of stem cells in the life cycle of the moss Physcomitrella patens. Curr. Opin. Plant Biol. 17, 13-21 (2014).

34. Szklarczyk, D. et al. STRING v11: protein-protein association networks with increased coverage, supporting functional discovery in genome-wide experimental datasets. Nucleic Acids Res. 47, D607-d613 (2019).

35. Frank, M. H. \& Scanlon, M. J. Cell-specific transcriptomic analyses of threedimensional shoot development in the moss Physcomitrella patens. Plant J. 83, 743-751 (2015).

36. Ishikawa, M. et al. Physcomitrella STEMIN transcription factor induces stem cell formation with epigenetic reprogramming. Nat. Plants 5, 681-690 (2019).

37. Turchi, L. et al. Arabidopsis HD-Zip II transcription factors control apical embryo development and meristem function. Development 140, 2118-2129 (2013).

38. Sakakibara, K. et al. WOX13-like genes are required for reprogramming of leaf and protoplast cells into stem cells in the moss Physcomitrella patens. Development 141, 1660-1670 (2014).

39. Posavec, M., Timinszky, G. \& Buschbeck, M. Macro domains as metabolite sensors on chromatin. Cell Mol. Life Sci. 70, 1509-1524 (2013).

40. Han, W., Li, X. \& Fu, X. The macro domain protein family: structure, functions, and their potential therapeutic implications. Mutat. Res 727, 86-103 (2011).

41. Zhang, H. et al. Arabidopsis PARG1 is the key factor promoting cell survival among the enzymes regulating post-translational poly(ADP-ribosyl)ation. Sci. Rep. 5, 15892 (2015).

42. Song, J., Keppler, B. D., Wise, R. R. \& Bent, A. F. PARP2 is the predominant poly(ADP-Ribose) polymerase in Arabidopsis DNA damage and immune responses. PLoS Genet. 11, e1005200 (2015).

43. Feng, B. et al. Protein poly(ADP-ribosyl)ation regulates arabidopsis immune gene expression and defense responses. PLoS Genet. 11, e1004936 (2015).

44. Berr, A. et al. Arabidopsis SET DOMAIN GROUP2 is required for H3K4 trimethylation and is crucial for both sporophyte and gametophyte development. Plant Cell 22, 3232-3248 (2010).

45. Chen, L. Q. et al. ATX3, ATX4, and ATX5 encode putative H3K4 methyltransferases and are critical for plant development. Plant Physiol. 174, 1795-1806 (2017)

46. Thorstensen, T., Grini, P. E. \& Aalen, R. B. SET domain proteins in plant development. Biochim. Biophys. Acta 1809, 407-420 (2011).

47. Baymaz, H. I., Karemaker, I. D. \& Vermeulen, M. Perspective on unraveling the versatility of 'co-repressor' complexes. Biochim. Biophys. Acta 1849, 1051-1056 (2015). 
48. Adams, G. E., Chandru, A. \& Cowley, S. M. Co-repressor, co-activator and general transcription factor: the many faces of the Sin3 histone deacetylase (HDAC) complex. Biochem. J. 475, 3921-3932 (2018).

49. Yaish, M. W., Peng, M. \& Rothstein, S. J. AtMBD9 modulates Arabidopsis development through the dual epigenetic pathways of DNA methylation and histone acetylation. Plant J. 59, 123-135 (2009).

50. Abplanalp, J. \& Hottiger, M. O. Cell fate regulation by chromatin ADPribosylation. Semin. Cell Dev. Biol. 63, 114-122 (2017).

51. Bannister, A. J. \& Kouzarides, T. Regulation of chromatin by histone modifications. Cell Res. 21, 381-395 (2011).

52. Verrier, L., Vandromme, M. \& Trouche, D. Histone demethylases in chromatin cross-talks. Biol. Cell 103, 381-401 (2011).

53. Birnbaum, K. D. \& Roudier, F. Epigenetic memory and cell fate reprogramming in plants. Regeneration 4, 15-20 (2017).

54. Briggs, A. G. \& Bent, A. F. Poly(ADP-ribosyl)ation in plants. Trends Plant Sci. 16, 372-380 (2011).

55. Feng, B., Liu, C., Shan, L. \& He, P. Protein ADP-ribosylation takes control in plant-bacterium interactions. PLoS Pathog. 12, e1005941 (2016).

56. Hoang, Q. T. et al. An actinoporin plays a key role in water stress in the moss Physcomitrella patens. N. Phytol. 184, 502-510 (2009).

57. Benito, B. \& Rodriguez-Navarro, A. Molecular cloning and characterization of a sodium-pump ATPase of the moss Physcomitrella patens. Plant J. 36, 382-389 (2003).

58. Guan, Y. et al. Gene refashioning through innovative shifting of reading frames in mosses. Nat. Commun. 9, 1555 (2018).

59. Van Etten, J. L. \& Dunigan, D. D. Chloroviruses: not your everyday plant virus. Trends Plant Sci. 17, 1-8 (2012).

60. Coy, S. R., Gann, E. R., Pound, H. L., Short, S. M. \& Wilhelm, S. W. Viruses of eukaryotic algae: diversity, methods for detection, and future directions. Viruses 10, 487 (2018).

61. Vlok, M., Gibbs, A. J. \& Suttle, C. A. Metagenomes of a freshwater charavirus from british columbia provide a window into ancient lineages of viruses. Viruses 11, 299 (2019).

62. Hoysted, G. A. et al. A mycorrhizal revolution. Curr. Opin. Plant Biol. 44, 1-6 (2018).

63. Li, M., Zhao, J., Tang, N., Sun, H. \& Huang, J. Horizontal gene transfer from bacteria and plants to the arbuscular mycorrhizal fungus Rhizophagus irregularis. Front. Plant Sci. 9, 701 (2018).

64. Martin, W. F. Too much eukaryote LGT. Bioessays 39, 1700115 (2017).

65. Leger, M. M., Eme, L., Stairs, C. W. \& Roger, A. J. Demystifying eukaryote lateral gene transfer (Response to Martin 2017 DOI: 10.1002/bies.201700115). Bioessays 40, 1700242 (2018).

66. Huang, J. Horizontal gene transfer in eukaryotes: the weak-link model. Bioessays 35, 868-875 (2013).

67. Soucy, S. M., Huang, J. \& Gogarten, J. P. Horizontal gene transfer: building the web of life. Nat. Rev. Genet. 16, 472-482 (2015).

68. Husnik, F. \& McCutcheon, J. P. Functional horizontal gene transfer from bacteria to eukaryotes. Nat. Rev. Microbiol. 16, 67-79 (2018).

69. Yue, J., Hu, X., Sun, H., Yang, Y. \& Huang, J. Widespread impact of horizontal gene transfer on plant colonization of land. Nat. Commun. 3, 1152 (2012).
70. Cove, D. J. et al. Transformation of the moss Physcomitrella patens using direct DNA uptake by protoplasts. Cold Spring Harb. Protoc. 2009, 5143 (2009).

\section{Acknowledgements}

We thank Stefan Rensing and Aizhong Liu for comments and suggestions to improve the manuscript, Jianqiang Wu for providing pM999 vector, Mitsuyasu Hasebe for providing pTN182 and pPOG1 vectors, Bojian Zhong for DNA samples of Spirogyra sp, and Yanxia Jia for chromosomal ploidy analyses. This work is funded in part by CAS Light of West China, National Natural Science Foundation of China (31970248), and the Second Tibetan Plateau Scientific Expedition and Research (STEP) Program (2019QZKK0502)

\section{Author contributions}

S.W. and J.H. conceived and designed the study. S.W., Y.G., Q.W., J.Z., G.S., X.H., and J.H. performed experiments and data analyses. M.P.R. and H.S. contributed to data interpretation and manuscript writing. S.W. and J.H. wrote the manuscript.

\section{Competing interests}

The authors declare no competing interests.

\section{Additional information}

Supplementary information is available for this paper at https://doi.org/10.1038/s41467 020-15967-6.

Correspondence and requests for materials should be addressed to H.S. or J.H.

Peer review information Nature Communications thanks the anonymous reviewers for their contribution to the peer review of this work. Peer review reports are available.

Reprints and permission information is available at http://www.nature.com/reprints

Publisher's note Springer Nature remains neutral with regard to jurisdictional claims in published maps and institutional affiliations.

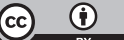

Open Access This article is licensed under a Creative Commons Attribution 4.0 International License, which permits use, sharing, adaptation, distribution and reproduction in any medium or format, as long as you give appropriate credit to the original author(s) and the source, provide a link to the Creative Commons license, and indicate if changes were made. The images or other third party material in this article are included in the article's Creative Commons license, unless indicated otherwise in a credit line to the material. If material is not included in the article's Creative Commons license and your intended use is not permitted by statutory regulation or exceeds the permitted use, you will need to obtain permission directly from the copyright holder. To view a copy of this license, visit http://creativecommons.org/ licenses/by/4.0/.

(C) The Author(s) 2020 